\title{
REMARKS ON NONOSCILLATION THEOREMS FOR A SECOND ORDER NONLINEAR DIFFERENTIAL EQUATION
}

JAMES S. W. WONG

\begin{abstract}
This paper proves two results concerning nonoscillation of solutions of the second order nonlinear differential equation

$$
y^{\prime \prime}+a(t)|y|^{\gamma} \operatorname{sgn} y=0, \quad \gamma>0,
$$

where $a(t)$ is positive, continuous and locally of bounded variation, and sgn $y$ denotes the sign of the function $y(t)$. Assume also that $a(t)$ satisfies $\int_{0}^{\infty} a^{-1}(s) d a_{+}(s)<\infty$. The main results are
\end{abstract}

TheOREM A. Let $0<\gamma<1$. If $\lim _{t \rightarrow \infty} t^{2} a(t)=0$, then $(\dagger)$ is nonascillatory.

THEOREM B. Let $\gamma>1$. If $\lim _{t \rightarrow \infty} t^{\gamma+1} a(t)=0$, then $(\dagger)$ is nonoscillatory.

1. Introduction. The purpose of this paper is to establish two nonoscillation theorems concerning solutions of the second order nonlinear equation

$$
y^{\prime \prime}+a(t)|y|^{\gamma} \operatorname{sgn} y=0, \quad \gamma>0,
$$

which were announced earlier [11, Theorem 4.14]. We are here concerned with the behaviour of solutions $y(t)$ of (1) on the half-infinite interval $[0, \infty)$; here prime denotes differentiation with respect to $t$ and $\operatorname{sgn} y$ denotes the sign of the function $y(t)$. It shall be assumed that the coefficient $a(t)$ is positive, continuous and locally of bounded variation on $[0, \infty)$. Under this assumption, it is known that all solutions of (1) can be continued throughout [0, $\infty$ ), see e.g. [3]. A nontrivial solution of (1) is said to be oscillatory if it has arbitrarily large zeros; otherwise it is said to be nonoscillatory, i.e. it is eventually of one sign. Equation (1) is called nonoscillatory if all solutions are nonoscillatory. We are here concerned with sufficient conditions on the coefficient $a(t)$ in order that (1) be nonoscillatory. From the equation itself, it is easy to see that if $y(t)$ is a solution so is $-y(t)$. It is also customary to distinguish equation (1) according to $\gamma>1$ and $\gamma<1$ and refer to them as the superlinear and sublinear equations respectively.

In [4], [5], we initiated a study of oscillation and nonoscillation results based upon a so-called "duality principle" between superlinear and sublinear equations, whereby results in one case would lead to the formulation of results in the other case. More specifically, in [4] we discuss results based upon monotonicity conditions on $a(t)$ whilst in [5] we study results based upon integral conditions. However, results in [5] are concerned only with oscillation of (1), and in particular its

Received by the editors August 27, 1980 and, in revised form, December 31, 1980.

AMS (MOS) subject classifications (1970). Primary 34C10, 34C15.

Key words and phrases. Second order, nonlinear, differential equation, oscillation. 
necessary and sufficient conditions. Here we shall explore this duality principle with regard to nonoscillation theorems for equation (1).

2. Statements of results. We summarize below a number of nonoscillation criteria for equation (1). For the linear equation, we have

(I) M. Bôcher [2], $\gamma=1, \int_{0}^{\infty} t a(t) d t<\infty$;

(II) A. Kneser [9], $\gamma=1$, lim $\sup _{t \rightarrow \infty} t^{2} a(t)<\frac{1}{4}$;

(III) W. Leighton [10], $\gamma=1, a(t)$ is nonincreasing,

$$
\int_{0}^{\infty} \sqrt{a(t)} d t<\infty
$$

In the superlinear case, also under the assumption that $a(t)$ is nonincreasing, we have

(IV) F. V. Atkinson [1], $\gamma>1, \int_{0}^{\infty} t^{\gamma} a(t) d t<\infty$.

For the sublinear case, again under assumption that $a(t)$ is nonincreasing, the result corresponding to the above is

(V) J. W. Heidel [7], $0<\gamma<1$, $\int_{0}^{\infty} \operatorname{ta}(t) d t<\infty$.

Heidel showed among other things that the monotonicity condition on $a(t)$ cannot be removed entirely when $\gamma \neq 1$. Thus, it is necessary to impose an additional condition on $a(t)$ in case of nonlinear equations.

The function $a(t)$ is said to be locally of bounded variation on $[0, \infty)$ if it is of bounded variation on each compact subinterval of $[0, \infty)$. If $a(t)$ is continuous and locally of bounded variation on $[0, \infty)$, then $a(t)$ admits the Jordan representation $a(t)=a_{+}(t)-a_{-}(t)$ where $a_{+}, a_{-}$are continuous nondecreasing functions of $t$. Gollwitzer [6] showed that the nonincreasing assumption on $a(t)$ can be weakened to that of

$$
\int_{0}^{\infty} \frac{d a_{+}(t)}{a(t)}<\infty
$$

More significantly, he applied techniques of Lyapunov functions coupled with Sturm's comparison theorem and obtained various extensions of (III), (IV) and (V). Thus, under the weaker assumption (2), he proved the following nonoscillation criteria for (1):

$$
\begin{aligned}
& \text { (VI) } \gamma>1,(2) \text { and } \int_{0}^{\infty} a(t)^{1 /(\gamma+1)} d t<\infty \\
& \text { (VII) } \gamma>1,(2), \int_{0}^{\infty} s^{\gamma-1} a(s) d s<\infty \text {, and } \\
& \qquad \lim _{t \rightarrow \infty} t \int_{t}^{\infty} s^{\gamma-1} a(s) d s=0 \\
& \text { (VIII) } \gamma>1,(2), \int_{0}^{\infty} a(s)^{2 /(\gamma+1)} d s<\infty \text {, and } \\
& \qquad \lim _{t \rightarrow \infty} t \int_{t}^{\infty} a(s)^{2 /(\gamma+1)} d s=0 \\
& \text { (IX) } 0<\gamma<1,(2), \int_{0}^{\infty} s^{\gamma} a(s) d s<\infty, \text { and } \\
& \qquad \lim _{t \rightarrow \infty} a(t)^{(\gamma-1) / 2} \int_{t}^{\infty} s^{\gamma} a(s) d s=0 .
\end{aligned}
$$


Using the "duality principle" introduced in our earlier work [4], [5], conditions for the oscillation and nonoscillation of the superlinear equation involving the parameter $\gamma$ can be translated into results for the sublinear equation by setting $\gamma=1$. This is evidenced here in Heidel's extension (V) of Atkinson's Theorem (IV), although methods of their proofs bear little resemblance.

Accordingly, Gollwitzer's results for the superlinear equation can be used to yield conjectures for probable nonoscillation criteria for the sublinear equation. Thus, condition (VI) would lead to

(X) $0<\gamma<1$, (2) and $\int_{0}^{\infty} \sqrt{a(t)} d t<\infty$; while conditions (VII) and (VIII) both lead to

(XI) $0<\gamma<1$, (2), $\int_{0}^{\infty} a(t) d t<\infty$, and

$$
\lim _{t \rightarrow \infty} t \int_{t}^{\infty} a(s) d s=0 .
$$

Indeed, we shall show that (X) follows from the following

TheOREM A. Let $0<\gamma<1$ and let $a(t)$ satisfy (2). Then,

$$
\lim _{t \rightarrow \infty} t^{2} a(t)=0
$$

implies that equation (1) is nonoscillatory.

On the other hand, Theorem A would follow from (XI) upon application of L'Hôpital's rule. Obviously, (V) follows from (XI) in the same way. We can also prove the superlinear counterpart of Theorem A, namely,

THEOREM B. Let $\gamma>1$ and let $a(t)$ satisfy (2). Then

$$
\lim _{t \rightarrow \infty} t^{\gamma+1} a(t)=0
$$

implies that equation (1) is nonoscillatory.

We note that Theorem B follows from (VII) by a simple application of L'Hôpital's rule. We shall proceed to show that (IV) follows from (VI) and (VI) in turn follows from Theorem B. Our results thus relate these existing nonoscillation criteria in an interesting way. Unfortunately, our methods fail to establish the validity of $(\mathrm{XI})$ as predicted under the "duality principle".

3. A technical lemma. The proofs of Theorem $A$ and other assertions depend on a result concerning integrals of functions satisfying (2), which may be of some interest in itself.

LEMma. Let $\mu$ be nonnegative, $\lambda$ be positive. Assume that $a(t)$ is positive, continuous, locally of bounded variation and satisfies (2). Then,

$$
\int_{0}^{\infty} s^{\mu} a^{\lambda}(s) d s<\infty
$$

implies

$$
\lim _{t \rightarrow \infty} t^{\mu+1} a^{\lambda}(t)=0
$$


Furthermore, if $0<\beta<\lambda$, then

$$
\lim _{t \rightarrow \infty} a^{\lambda-\beta}(t) t^{\mu+1}=0
$$

implies

$$
\lim _{t \rightarrow \infty} a^{-\beta}(t) \int_{t}^{\infty} s^{\mu} a^{\lambda}(s) d s=0
$$

Proof. The following identify follows from a simple integration by parts:

$$
\int_{0}^{t} s^{\mu} a^{\lambda}(s) d s=\frac{t^{\mu+1}}{\mu+1} a^{\lambda}(t)-\frac{\lambda}{\mu+1} \int_{0}^{t} s^{\mu+1} a^{\lambda-1}(s) d a(s) .
$$

Let $\alpha(t)=t^{\mu+1} a^{\lambda}(t)$. It follows from (9) that $\alpha(t)$ satisfies the inequality

$$
\alpha(t) \leqslant B(\mu+1)+\lambda \int_{0}^{t} \alpha(s) \frac{d a_{+}(s)}{a(s)},
$$

where $B=\int_{0}^{\infty} s^{\mu} a^{\lambda}(s) d s$. An application of Gronwall's inequality to (10) yields that $\alpha(t)$ is bounded on account of (2). Since $a(t)$ is positive, we obtain also from (9) the following estimate:

$$
\frac{\lambda}{\mu+1} \int_{0}^{t} \alpha(s) \frac{d a_{-}(s)}{a(s)} \leqslant \int_{0}^{t} s^{\mu} a^{\lambda}(s) d s+\frac{\lambda}{\mu+1} \int_{0}^{t} \alpha(s) \frac{d a_{+}(s)}{a(s)} .
$$

As $\alpha(t)$ is bounded and $a(t)$ satisfies (2), it follows from (5) and (11) that the integral on the left has a finite limit as $t \rightarrow \infty$; hence $\int_{0}^{\infty} s^{\mu+1} a^{\lambda-1}(s) d a(s)$ exists and is finite. Returning to (9), we deduce therefore that $\lim _{t \rightarrow \infty} t^{\mu+1} a^{\lambda}(t)$ exists and is nonnegative. However, if such a limit is positive, then $t^{\mu} a^{\lambda}(t) \geqslant \varepsilon / t$ for some $\varepsilon>0$ and for all sufficiently large $t$, which would contradict assumption (5), proving (6).

We now assume (7) holds. Since $0<\beta<\lambda$, it is easy to verify that (7) implies (5). Thus, we can obtain the following identity upon integration by parts:

$$
\int_{t}^{\infty} s^{\mu} a^{\lambda}(s) d s=-\frac{t^{\mu+1}}{\mu+1} a^{\lambda}(t)-\frac{\lambda}{\mu+1} \int_{t}^{\infty} s^{\mu+1} a^{\lambda-1}(s) d a(s) .
$$

Thus, to prove (8), it is sufficient to show that

$$
\lim _{t \rightarrow \infty} a^{-\beta}(t) \int_{t}^{\infty} s^{\mu+1} a^{\lambda-1}(s) d a_{-}(s)=0 .
$$

Before proving (13), we first note that if $a(t)$ satisfies (2), then for all $t>0$, we have

$$
\sup _{s>t} \frac{a(s)}{a(t)} \leqslant K<\infty
$$

where the constant $K$ is independent of $t$. Note that

$$
\log \frac{a(s)}{a(t)}=\int_{t}^{s} \frac{d a(\tau)}{a(\tau)} \leqslant \int_{t}^{s} \frac{d a_{+}(\tau)}{a(\tau)} \leqslant \int_{0}^{\infty} \frac{d a_{+}(s)}{a(s)},
$$

from which (14) follows from (2) at once. To prove (13) we fix any $\varepsilon>0$, and choose $T \geqslant 0$ such that for all $t \geqslant T$,

$$
a^{\lambda-\beta}(t) t^{\mu+1} \leqslant \varepsilon \beta / 2,
$$


which is possible by (7). Using (15), we can estimate the integral in (13) as follows:

$$
\begin{aligned}
\int_{t}^{\infty} s^{\mu+1} a^{\lambda-1}(s) d a_{-}(s) & \leqslant \frac{\varepsilon \beta}{2}\left\{\int_{t}^{\infty} a^{\beta-1}(s)\left(d a_{+}(s)-d a(s)\right)\right\} \\
& \leqslant \frac{\varepsilon \beta}{2} \int_{t}^{\infty} a^{\beta-1}(s) d a_{+}(s)+\frac{\varepsilon}{2} a^{\beta}(t)
\end{aligned}
$$

The integral on the right of (16) can now be estimated by

$$
\int_{t}^{\infty} a^{\beta-1}(s) d a_{+}(s) \leqslant K^{\beta} a^{\beta}(t) \int_{t}^{\infty} \frac{d a_{+}(s)}{a(s)} .
$$

In view of (2), we can choose $T_{1} \geqslant T$ so that

$$
\beta K^{\beta} \int_{t}^{\infty} \frac{d a_{+}(s)}{a(s)} \leqslant 1, \text { for } t \geqslant T_{1}
$$

Combining (17), (18) in (16) and noting that $\varepsilon>0$ is aribtrary, we obtain the desired conclusion (13). This completes the proof of the lemma.

4. Remarks. We first note that condition (3) can be rewritten as

$$
\lim _{t \rightarrow \infty} a(t)^{(\gamma+1) / 2} t^{\gamma+1}=0 .
$$

Setting $\lambda=1, \beta=(1-\gamma) / 2$, and $\mu=\gamma$ in (7), we find the above expression. Applying the lemma just proved, we see that (8) reduces to (IX), so equation (1) is nonoscillatory.

We shall now show that $(X)$ follows from the improved version of $(V)$, i.e. when $a(t)$ satisfies the weaker assumption (2). Note that for any $\gamma>0$,

$$
\int_{0}^{\infty} a(t)^{1 /(\gamma+1)} d t<\infty \Rightarrow \int_{0}^{\infty} s^{\gamma} a(s) d s<\infty
$$

Take $\lambda=1 /(\gamma+1), \mu=0$ in (5); then (6) becomes

$$
\lim _{t \rightarrow \infty} \operatorname{ta}(t)^{1 /(\gamma+1)}=0
$$

which implies that there exists a constant $M$ such that $\left|\operatorname{ta}(t)^{1 /(\gamma+1)}\right|<M$. Consequently, $\left|t^{\gamma} a(t)^{\gamma /(\gamma+1)}\right| \leqslant M^{\gamma}$. Multiplying by $a(t)^{1 /(\gamma+1)}$ on both sides, we have $\left|t^{\gamma} a(t)\right| \leqslant M^{\gamma} a(t)^{1 /(\gamma+1)}$, yielding the desired conclusion (19). Note that (19) shows, first of all, (III) follows from (I) and is indeed a special case of a much older result. It also shows that (VI) follows from the improved version of (IV). Taking $\gamma=1$ in (19), we easily see that (X) follows from (V), and setting $\mu=\lambda=1$ in (5), it follows that (6) becomes (3), so (V) follows from Theorem A. If we set $\mu=\gamma, \lambda=1$ in (5), then (6) becomes (4), so (IV) follows from Theorem B.

ACKNOWLEDGEMENT. The author is grateful to his referees for pointing out an error in the original manuscript purporting that (XI) follows from Theorem A while the reverse is true. 


\section{REFERENCES}

1. F. V. Atkinson, On second order nonlinear oscillations, Pacific J. Math. 5 (1955), 643-647.

2. M. Bôcher, The theorems of oscillation of Sturm and Klein. I, II, Bull. Amer. Math. Soc. 4 (1897/8), $295-313 ; 365-376$.

3. C. V. Coffman and D. F. Ullrich, On the continuation of solutions of a certain nonlinear differential equation, Monatsh. Math. 71 (1967), 385-392.

4. C. V. Coffman and J. S. W. Wong, Oscillation and nonoscillation of solutions of generalized Emden-Fowler equations, Trans. Amer. Math. Soc. 167 (1972), 399-434.

5. Oscillation and nonoscillation theorems for second order ordinary differential equations, Funkcial. Ekvac. 15 (1972), 119-130.

6. M. E. Gollwitzer, Nonoscillation theorems for a nonlinear differential equation, Proc. Amer. Math. Soc. 26 (1970), 78-84.

7. J. W. Heidel, A nonoscillation theorem for a nonlinear second order differential equation, Proc. Amer. Math. Soc. 22 (1969), 485-488.

8. D. V. Izyumova and I. T. Kiguradze, Some remarks on the solutions of the equation $u^{\prime \prime}+a(t) f(u)$ = 0, Differencial'nye Uravnenija 4 (1968), 589-605. (Russian)

9. A. Kneser, Untersuchung über die reellen Nullstellen der Integrale linear Differentialgleichungen, Math. Ann. 42 (1893), 409-435.

10. W. Leighton, On self-adjoint differential equation of second order, J. London Math. Soc. 27 (1952), 37-47.

11. J. S. W. Wong, On the generalized Emden-Fowler equation, SIAM Rev. 17 (1975), 339-360.

China Dyeing Works, LTD., 833 Swire House, Hong Kong 